\title{
Atypical aortic arch branching variants: A novel marker for thoracic aortic disease
}

\author{
Julia Dumfarth, MD, ${ }^{\mathrm{a}}$ Alan S. Chou, BA, ${ }^{\mathrm{a}}$ Bulat A. Ziganshin, MD,,${ }^{\mathrm{a} b}$ Rohan Bhandari, MS, ${ }^{\mathrm{a}}$ \\ Sven Peterss, MD, ${ }^{\mathrm{a}}$ Maryann Tranquilli, RN, ${ }^{\mathrm{a}}$ Hamid Mojibian, MD, ${ }^{\mathrm{c}}$ Hai Fang, $\mathrm{PhD},{ }^{\mathrm{d}}$ \\ John A. Rizzo, PhD, ${ }^{e}$ and John A. Elefteriades, $\mathrm{MD}^{\mathrm{a}}$
}

\begin{abstract}
Objective: To examine the potential of aortic arch variants, specifically bovine aortic arch, isolated left vertebral artery, and aberrant right subclavian artery, as markers for thoracic aortic disease (TAD).

Methods: We screened imaging data of 556 patients undergoing surgery due to TAD for presence of aortic arch variations. Demographic data were collected during chart review and compared with a historical control group of 4617 patients.

Results: Out of 556 patients with TAD, 33.5\% (186 patients) demonstrated anomalies of the aortic arch, compared with $18.2 \%$ in the control group $(P<.001)$. Three hundred seventy $(66.5 \%)$ had no anomaly of the aortic arch. Bovine aortic arch emerged as the most common anomalous branch pattern with a prevalence of $24.6 \%(n=137)$. Thirty-five patients $(6.3 \%)$ had an isolated left vertebral artery, and 10 patients $(1.8 \%)$ had an aberrant right subclavian artery. When compared with the control group, all 3 arch variations showed significant higher prevalence in patients with TAD $(P<.001)$. Patients with aortic aneurysms and anomalous branch patterns had hypertension less frequently $(73.5 \%$ vs $81.8 \% ; P=.048)$, but had a higher rate of bicuspid aortic valve $(40.8 \%$ vs $30.6 \% ; P=.042)$ when compared with patients with aneurysms but normal aortic arch anatomy. Patients with aortic branch variations were significantly younger $(58.6 \pm 13.7$ years vs $62.4 \pm 12.9$ years; $P=.002)$ and needed intervention for the aortic arch more frequently than patients with normal arch anatomy ( $46 \%$ vs $34.6 \% ; P=.023)$.
\end{abstract}

Conclusions: Aortic arch variations are significantly more common in patients with TAD than in the general population. Atypical branching variants may warrant consideration as potential anatomic markers for future development of TAD. (J Thorac Cardiovasc Surg 2015;149:1586-92)

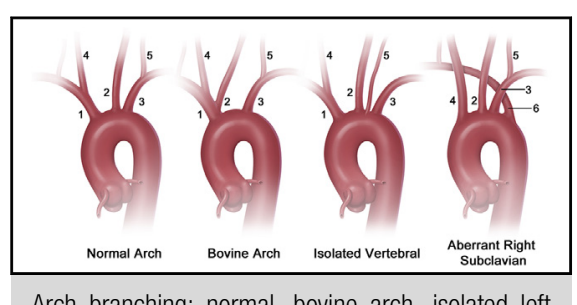

Arch branching: normal, bovine arch, isolated left vertebral artery, aberrant right subclavian artery.

Central Message

Variations of the aortic arch are more common in patients with thoracic aortic disease. Therefore, atypical branching variants should no longer be considered clinically irrelevant. Instead they may warrant consideration as potential anatomic markers for future development of thoracic aortic disease.

\section{Perspective}

Prevalence of aortic arch variations is elevated in patients with thoracic aortic disease. Patients with aortic arch variations are younger and need more extensive surgical procedures than patients with normal aortic arch anatomy. Arch anomalies can considerably influence perfusion strategies as well as surgical treatment. Therefore, clinicians should pay special attention to imaging studies and carefully evaluate the anatomy of the aortic arch in all patients with thoracic aortic disease. Different branching patterns should no longer be considered benign variations but rather as potential anatomic markers or risk factors for future thoracic aortic disease.

See Editorial Commentary page 1593.
From the Aortic Institute at Yale-New Haven Hospital, ${ }^{\mathrm{a}}$ and Department of Radiology, ${ }^{\mathrm{c}}$ Yale University School of Medicine, New Haven, Conn; Department of Surgical Diseases \# 2, ${ }^{\text {b }}$ Kazan State Medical University, Kazan, Russia; China Center for Health Development Studies, ${ }^{\mathrm{d}}$ Peking University, Beijing, China; and Department of Economics and Department of Preventive Medicine, ${ }^{\mathrm{e}}$ Stony Brook University, Stony Brook, NY.

J.D. and A.S.C. contributed equally to this work.

Read at The American Association for Thoracic Surgery Aortic Symposium, New York, New York, April 24-25, 2014

Received for publication Nov 5, 2014; revisions received Jan 27, 2015; accepted for publication Feb 9, 2015; available ahead of print March 20, 2015.

Address for reprints: John A. Elefteriades, MD, 789 Howard Ave, Clinic Building CB317, New Haven, CT 06519 (E-mail: john.elefteriades@yale.edu).

0022-5223/\$36.00

Copyright (c) 2015 by The American Association for Thoracic Surgery

http://dx.doi.org/10.1016/j.jtcvs.2015.02.019
According to the latest report of the Centers of Disease Control and Prevention, aortic aneurysms are the 18th leading cause of death in the United States. ${ }^{1}$ Because thoracic aneurysms are usually asymptomatic, identification of thoracic aortic aneurysms and prevention of complications of the disease can be challenging for physicians. Timely detection is crucial. Identification of associated conditions and biomarkers of aortic disease would enhance diagnosis. A bicuspid aortic valve and a strong family history of aortic disease are well-known risk factors for thoracic aortic disease (TAD), as is the presence of intracranial aneurysm..$^{2-4}$ The significance of 


\section{Abbreviations and Acronyms \\ LSA $=$ left subclavian artery \\ $\mathrm{TAD}=$ thoracic aortic disease}

aortic arch anomalies as associates of aneurysm disease is largely unexplored.

Anatomic variations of the aortic arch and its vessels are common in the general population. ${ }^{5-8}$ They are usually considered clinically insignificant or benign variants and are commonly asymptomatic. To date 5 large imaging studies have been published depicting the frequency of the 3 well-defined variations (Figure 1$)^{5-9}$ :

1) Bovine aortic arch: A common origin of the left common carotid artery and the brachiocephalic artery at the aortic arch, or less commonly a variation in which the left common carotid artery arises directly from the brachiocephalic artery;

2) Isolated left vertebral artery: A branching variant in which the left vertebral artery originates directly from the aortic arch; and

3) Aberrant right subclavian artery: Right subclavian artery originating directly from the aortic arch as a fourth branch, typically distal to the left subclavian artery (LSA).

In recent years the presence of a bovine aortic arch-the most common anatomic variation-was studied extensively in terms of clinical implications in patients with TAD. Based on the association of a bovine aortic arch with higher growth rates of the thoracic aorta and a higher prevalence in patients with TAD compared with the general population, this arch anomaly is currently viewed as a marker or risk factor for thoracic aortic aneurysms and their lifethreatening complications. ${ }^{10,11}$

We aimed to describe the prevalence of aortic arch variations in patients with known TAD and their influence on the natural history of the disease or their potential role as an independent risk factor.

\section{PATIENTS AND METHODS Patient Population}

TAD was defined as either aneurysmal dilation of the thoracic aorta (diameter $\geq 4.0 \mathrm{~cm}$ ) or presence of aortic dissection, intramural hematoma, or rupture. Our usual criterion for surgery is $5 \mathrm{~cm}$ for the ascending aorta; however, selected patients may undergo operation if the aorta is smaller; for example, in case of symptoms, rapid growth, high aortic size index, or positive genetic test for early dissection syndrome. ${ }^{12}$

Our study was approved by the Institutional Review Board of Yale University. We turned a special eye toward isolated left vertebral artery and aberrant subclavian artery. We examined the records of 590 consecutive patients undergoing surgery TAD identified through the database of the Aortic Institute at Yale-New Haven Hospital. We chose the years 2008 to 2013 for our review. In evaluating the computed tomography scans, we took this opportunity to add to our prior published information on bovine aortic arch. ${ }^{10}$ The time period of this review overlaps by 1.5 years with our prior report on bovine arch, but it includes 321 unique new cases studied and 83 unique new bovine aortic arch patients above and beyond our prior report, as well as our first-ever analysis of isolated left vertebral and aberrant subclavian artery.

Due to lack of appropriate imaging data, 34 patients were excluded. In total 556 patients were included for further analysis (mean age at presentation, $58.9 \pm 13.8$ years; range, $6-85$ years; $69.2 \%$ male). Patients were divided into 2 groups based on the underlying disease: aortic aneurysms $(\mathrm{n}=432 ; 77.7 \%)$ and acute aortic syndromes $(\mathrm{n}=124 ; 22.9 \%)-$ aortic dissections (type $\mathrm{A}, \mathrm{n}=96[17.3 \%]$ and type $\mathrm{B}, \mathrm{n}=23[4.1 \%]$ ), intramural hematoma $[\mathrm{n}=2]$, and aortic rupture $[\mathrm{n}=3]$.

We used all large-scale aortic imaging studies depicting different branch patterns in the general population to create a historical control population of 4617 patients. ${ }^{5-8}$

A study by Berko and colleagues ${ }^{9}$ examined images of patients with suspected pulmonary embolism or aortic dissection. We believed this might not reflect a healthy general patient population and might carry confounding factors if used as a control cohort. For this reason data from this imaging study were excluded from our control group.

In addition to imaging data, patient demographic characteristics as well as risk factors, surgical procedures, and details on the underlying aortic disease as well as morphology of the aortic valve were collected during the chart review. Furthermore, we calculated age at the time of surgery and age at presentation-defined as the time when patients were first seen by a cardiologist or cardiac surgeon due to aortic disease.

\section{Definition of Aortic Branching Variants}

Prevalence of aortic branching variants was determined by evaluating radiographic images (computed tomography or magnetic resonance imaging scans). Axial images were available for all patients with TAD; coronal, sagittal, and 3-dimensional reconstruction images were used for additional confirmation when available.

The following radiologic criteria were used for 3 different aortic arch patterns:

1. The presence of bovine aortic arch was determined if the brachiocephalic artery and left common carotid artery had their separation point cranial to the plane of the greater curvature of the aortic arch.

2. An isolated left vertebral artery was noted as a left vertebral artery arising directly from the aortic arch, either proximal or distal to the LSA.

3. An aberrant right subclavian artery was defined as a fourth vessel arising distal to the LSA and crossing to the posterior of the mediastinum toward the upper right extremity.

These 3 most common anatomic variations are illustrated in Figure 1.

\section{Statistical Analysis}

Statistical analysis was performed using SPSS version 21.0 (IBM-SPSS Inc, Armonk, NY). Categorical variables are expressed as frequency distributions and percentages; continuous variables are expressed as mean \pm standard deviation. The $\chi^{2}$ test or Fischer exact test was used for categorical variables and the Student $t$ test or Mann-Whitney $U$ test was used for continuous variables. ${ }^{13}$

To highlight the influence of single-branch patterns, all tests were performed comparing patients with specific anatomic variations to normal aortic arch patterns. For comparison of the TAD patient cohort to the healthy general population, data from 4 large imaging studies were summated and used as a historical control group throughout our analysis. Details on the control group are given in Table 1.

For comparison of comorbidities in different patient cohorts the $\chi^{2}$ test was performed. Based on the significant association in patients with aortic aneurysms, data were taken into a multivariate analysis. Due to the small sample size in the subgroups (especially patients with an aberrant 


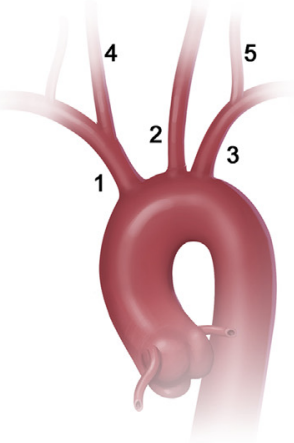

Normal Arch

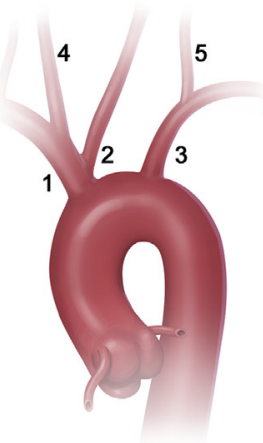

Bovine Arch

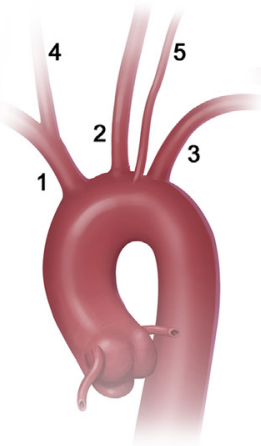

Isolated Vertebral

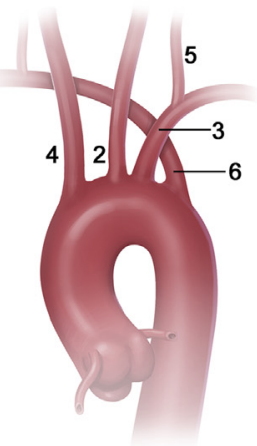

Aberrant Right Subclavian

FIGURE 1. Aortic arch branching patterns: normal branching variant of the aortic arch, bovine aortic arch variant, isolated left vertebral artery variant, and aberrant right subclavian artery. 1 = Brachiocephalic artery. 2 = Left common carotid artery. $3=$ Left subclavian artery. $4=$ Right common carotid artery. $5=$ Left vertebral artery. $6=$ Aberrant right subclavian artery.

subclavian artery) all patients with anatomic variations were clustered together in the multivariate model.

Patients displaying a combination of anatomic variations (ie, $>1$ ) were included in calculations on the total number but excluded when isolated branch patterns were taken for further analysis $(n=4 ; 0.9 \%)$.

\section{RESULTS}

\section{Prevalence of Aortic Arch Branching Variants}

Overall anomalies. Out of 556 patients undergoing surgery $66.5 \%(\mathrm{n}=370)$ (mean age, $62.4 \pm 12.9$ years; $68.6 \%$ male) presented with a normal branching pattern of the aortic arch. This represented a significantly lower prevalence when compared with the general population $(66.5 \%$ vs $81 \% ; P<.001)$. Alternatively stated, $33.5 \%$ of the TAD group had arch anomalies, compared with $18.2 \%$ in the control group (Figure 2).

Bovine aortic arch. The bovine aortic arch emerged as the most common branch variation in the TAD group as well as in the control group. Prevalence of isolated bovine aortic arch in the TAD cohort was $24.6 \%(n=137)$. Four patients revealed additional arch anomalies concomitant with a bovine aortic arch (bovine aortic arch + isolated left vertebral artery in $0.5 \%[\mathrm{n}=3]$ and bovine aortic arch + aberrant right subclavian artery in $0.2 \%[n=1]$ ). Isolated bovine aortic arch was more common in patients with TAD when compared with controls $(24.6 \%$ vs $14 \%$; $P<.001)$.

Isolated left vertebral artery. Isolated left vertebral artery was the second most common arch pattern in the general population. Thirty-five patients $(6.3 \%)$ of the TAD group presented with an isolated left vertebral artery, revealing a significantly higher prevalence when compared with the control group $(6.3 \%$ vs $3.4 \% ; P<.001)$. The origin of the isolated left vertebral artery in the aortic arch was located between the left carotid artery and the LSA in 34 out of 35 patients $(97.1 \%)$. In the remaining 1 patient, the isolated left vertebral artery arose from the arch distal to the LSA. Figure 3 shows the 2 different locations of the origin of the isolated left vertebral artery in the aortic arch. Aberrant right subclavian artery. An aberrant right subclavian artery was detected in only 10 patients $(1.8 \%)$ but was still more common in the TAD population when compared with the control group $(1.8 \%$ vs $0.6 \% ; P<.001)$. All anomalies. Based on the data given in our study, prevalence of every single-branch pattern was significantly higher when compared with any of the published data on the general population.

Aneurysm versus acute aortic syndrome. Patients were stratified into 2 groups based on their underlying disease-aneurysm or acute aortic syndrome (ie, intramural hematoma, dissection, or rupture). Distribution of arch patterns did not differ between the 2 groups (Table 2).

\section{Patient Demographics and Risk Factors for Aortic Disease}

Risk factor analysis comparing anomalous branch patterns with patients without arch anomalies revealed no

TABLE 1. Control groups

\begin{tabular}{|c|c|c|c|c|c|c|c|c|c|c|c|}
\hline Pattern & $\begin{array}{c}\text { Yale data } \\
\text { (TAD) }\end{array}$ & Celikyay $^{5}$ & $\begin{array}{c}P \\
\text { value }^{*}\end{array}$ & Muller $^{7}$ & $\begin{array}{c}P \\
\text { value* }\end{array}$ & Jakanani $^{6}$ & $\begin{array}{c}P \\
\text { value* }\end{array}$ & Natsis $^{8}$ & $\begin{array}{c}P \\
\text { value* }\end{array}$ & $\begin{array}{c}\text { Total } \\
\text { controls }\end{array}$ & $\begin{array}{c}P \\
\text { value* }\end{array}$ \\
\hline Normal pattern & $370(66.5)$ & $845(74.4)$ & $<.001$ & $1763(86.7)$ & $<.001$ & $643(74)$ & $<.001$ & $527(83)$ & $<.001$ & $3778(81.8)$ & $<.001$ \\
\hline Bovine aortic arch & $137(24.6)$ & $240(21.1)$ & .03 & $163(8)$ & $<.001$ & $155(18)$ & .001 & $96(15)$ & $<.001$ & $654(14.2)$ & $<.001$ \\
\hline Isolated left vertebral artery & $35(6.3)$ & $34(3)$ & $<.001$ & $86(4.2)$ & .001 & $32(3.7)$ & .01 & $5(0.8)$ & $<.001$ & $157(3.4)$ & $<.001$ \\
\hline Aberrant right subclavian artery & $10(1.8)$ & $4(0.4)$ & $<.001$ & $20(1)$ & .022 & $4(0.5)$ & .007 & $0(0)$ & $<.001$ & $28(0.6)$ & $<.001$ \\
\hline
\end{tabular}

Values are presented as $\mathrm{n}(\%)$. TAD, Thoracic aortic disease. ${ }^{*}$ Calculated versus Yale data. 


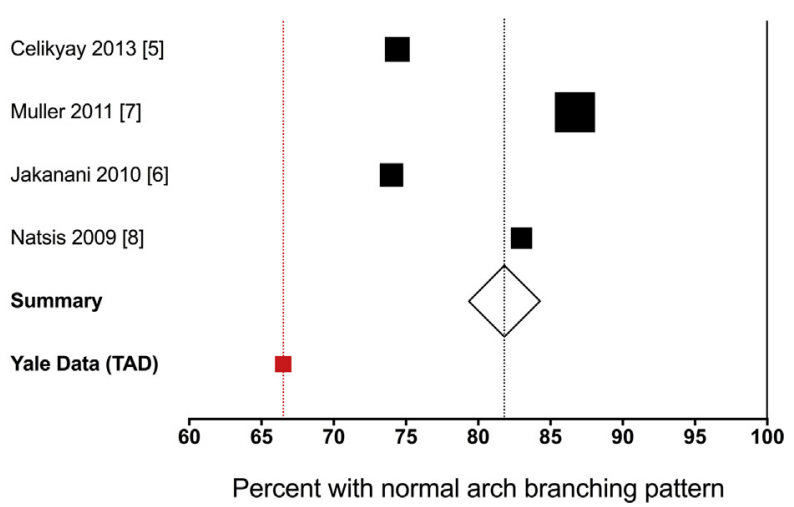

FIGURE 2. Modified Forest plot of percent of patients with normal arch branching pattern. Note the much lower frequency of normal branching pattern in the patients with thoracic aortic disease (TAD) compared with the normal populations. This is a visual representation of the data from Table 1 . This was done by depicting the data from each study by a square located at the proper percent line for each study, and with a weighted square size for the number of patients studied. The diamond represents the average of all 4 studies of the normal population. The Yale study of the TAD population is represented by the red square. The confidence limits for the studies from the literature were not available, so no formal Forest plot statistical calculations could be done. The applicable statistics are those from Table 1.

difference in the distribution of hypertension, hyperlipidemia, history of smoking, diabetes, or presence of bicuspid aortic valve (Table 3).

Patients displaying anomalous branching were younger at time of initial presentation $(56.7 \pm 14$ years vs $60.1 \pm 13.7$ years; $P=.007)$ as well as at time of surgery $(58.6 \pm 13.7$ years vs $62.4 \pm 12.9$ years; $P=.002$ ). Subgroup analysis based on type of arch patterns confirmed this observation in patients with bovine aortic arch $(56.7 \pm 13.8$ years vs $60.1 \pm 13.7$ years; $P=.013$ for age at first presentation; $58.5 \pm 13.5$ years vs $62.4 \pm 12.9$ years; $P=.003$ for age at surgery).
Patients with aortic aneurysms. In patients with anomalous arch branching, hypertension was less frequent $(73.5 \%$ vs $81.8 \% ; P=.048)$ than in our patients with a normal branch pattern. Prevalence of a bicuspid aortic valve was significantly higher when compared with patients with normal arch anatomy ( $40.8 \%$ vs $30.6 \% ; P=.042)$. There was a clear gender difference in patients with an isolated left vertebral artery when compared with patients without arch anomalies; isolated left vertebral artery affected predominantly male patients $(92.3 \%$ vs $68.8 \%$ in patients with normal branch patterns; $P=.012$ ). Again, patients with congenital arch anomalies were significantly younger at first presentation ( $56.6 \pm 14$ years vs $59.8 \pm 13.5$ years; $P=.023$ ) as well as at the time of surgery $(58.8 \pm 13.7$ years vs $62.3 \pm 12.8$ years; $P=.008$ ) (Table 4). Multivariate analysis identified presence of bicuspid aortic valve as the only independent risk factor for TAD in patients with anomalous arch branching (odds ratio, $1.519 ; 95 \%$ confidence interval, $1.02-2.308 ; P=.049$ ) (Table 5).

Patients with acute aortic syndrome. Interestingly, neither risk factors nor age at presentation or surgery differed in patients with acute aortic syndrome. Mean diameter of the ascending aorta at the level of the pulmonary artery bifurcation in patients with type $\mathrm{A}$ aortic dissection was $5 \pm 1.4 \mathrm{~cm}$. Patients undergoing surgery for type B aortic dissection, impending rupture, or intramural hematoma of the descending aorta had a mean diameter of $5.53 \pm 1.5$ $\mathrm{cm}$. There was no significant difference in aortic size between patients with arch anomalies and normal arch patterns except a trend toward smaller aortic diameter in patients with bovine aortic arch and acute type A dissection $(4.8 \pm 1.8 \mathrm{~cm}$ vs $5.1 \pm 1.4 \mathrm{~cm}$ normal arch anatomy).

\section{Effect of Branching Patterns on Surgical Procedures}

All 556 patients underwent surgery due to the documented aortic disease. Two hundred seventy-six patients
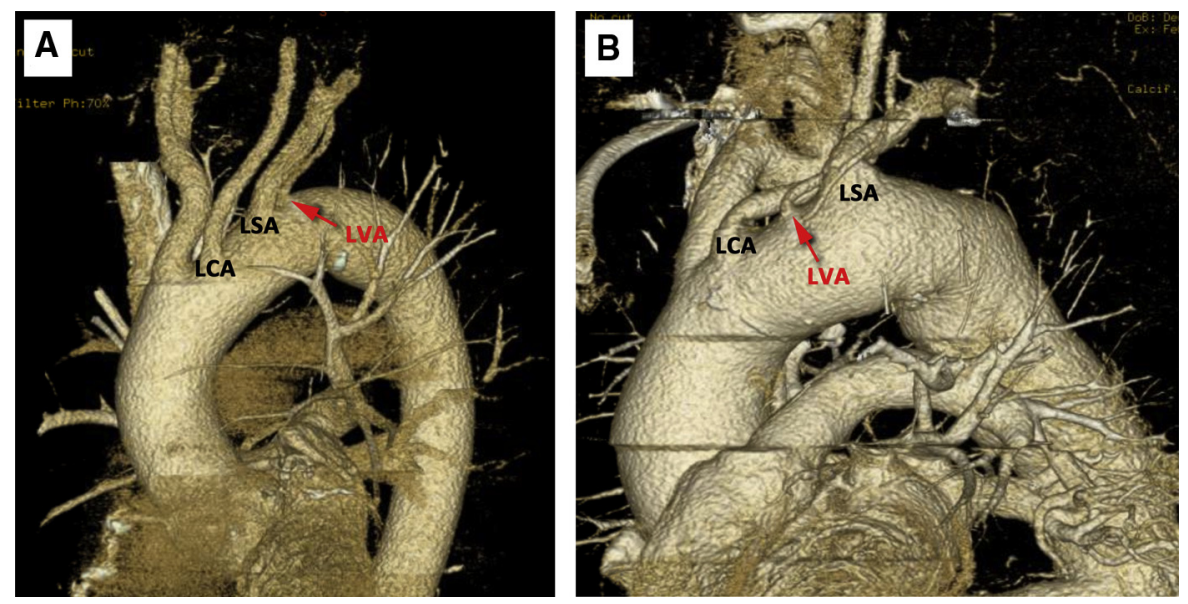

FIGURE 3. Different location of the origin of isolated left vertebral artery (LVA) (red arrow). A, LVA originating directly from the aortic arch distal to the left subclavian artery $(L S A)$. B, LVA originating from the aortic arch between the left common carotid artery $(L C A)$ and the LSA. 
TABLE 2. Distribution of arch patterns based on underlying disease

\begin{tabular}{lccc}
\hline \multicolumn{1}{c}{ Pattern } & $\begin{array}{c}\text { Aneurysm } \\
(\mathbf{n = 4 3 2 )}\end{array}$ & $\begin{array}{c}\text { Acute aortic } \\
\text { syndrome }(\mathbf{n = 1 2 4 )})\end{array}$ & $\begin{array}{c}\boldsymbol{P} \\
\text { value }\end{array}$ \\
\hline Normal pattern & $285(66)$ & $85(68.5)$ & .666 \\
Bovine aortic arch & $109(25.2)$ & $28(22.6)$ & .640 \\
Isolated left vertebral artery & $26(6)$ & $9(7.3)$ & .546 \\
Aberrant right subclavian artery & $9(2.1)$ & $1(0.8)$ & .470 \\
\hline Values are presented as n $(\%)$. & & &
\end{tabular}

Values are presented as $\mathrm{n}(\%)$.

underwent ascending replacement $(49.6 \%)$ and 220 underwent ascending plus hemiarch or arch replacement $(39.6 \%)$. Isolated arch replacement was performed in 5 patients $(0.9 \%)$. Descending replacement, including distal arch replacement, was done 6 times $(1.1 \%)$. Twenty-six patients $(4.7 \%)$ underwent descending and 23 patients $(4.1 \%)$ underwent thoracoabdominal replacement. Rate of intervention of the aortic arch (hemiarch or total arch) was higher in patients displaying abnormal branch patterns compared with patients with normal aortic arch anatomy $(45.7 \%$ vs $36.3 \% ; P=.034)$ (Table 6$)$.

\section{DISCUSSION}

Variations of aortic arch patterns are well described in the general population. Due to the fact that variations of the aortic arch are usually asymptomatic, they are most commonly discovered incidentally.

In recent years there has been growing awareness of the presence of arch anomalies when it comes to planning of surgical or interventional procedures in the chest or neck. The bovine aortic arch - the most common variation-has gained importance due to the newly recognized association of this anomaly with aortic disease. The bovine aortic arch is currently discussed as a new anatomic risk factor or biomarker for aortic disease. ${ }^{10,11,14}$ Although great effort has recently been put into analysis of frequencies of different arch patterns in the general population, very little is known about different branch patterns in patients with aortic disease.

In terms of potential associations of aortic arch anomalies and TAD, 2 case reports describe the presence of a
Kommerell diverticulum in patients with acute aortic dissections. ${ }^{15,16}$ Stone and colleagues ${ }^{17}$ addressed treatment options of the right aberrant subclavian artery in a series of 24 patients. They concluded that the presence of a Kommerell diverticulum in these patients correlated with the need for intervention, whereas the presence of aberrant right subclavian artery alone could be safely followed medically.

The association of innominate artery injury in blunt trauma and bovine aortic arch was described in reports by Moise and colleagues ${ }^{18}$ and Wells and colleagues. ${ }^{19}$ Based on the anatomic variant of a bovine aortic arch, the number of fixation points are decreased. Wells and colleagues ${ }^{19}$ postulate that energy forces in blunt trauma are concentrated on the innominate artery takeoff, resulting in tears or transections.

Recently Wanamaker and colleagues ${ }^{11}$ published a study on arch anomalies in patients with thoracic aortic dissections. This study showed a higher prevalence of arch anomalies in patients with thoracic aortic dissections when compared with controls. Detailed analysis on different patterns revealed bovine aortic arch as the most common arch variation but failed to show elevated prevalence in different arch anomalies other than bovine aortic arch.

Total prevalence of aortic arch anomalies was elevated in our study in patients with TAD when compared with controls. We were able to show higher rates of every single branch pattern in our population with aortic disease. We previously demonstrated higher prevalence of bovine aortic arch in patients with TAD and elevated aortic growth rates in patients with bovine aortic arch and TAD compared with those without bovine aortic arch. ${ }^{10}$ As a result of our current study, we believe that aortic arch variations in general should be considered as new risk factors for aortic disease. Biomarker in the global sense refers to a biologic indicator of the presence of disease; in this sense, aortic arch anomalies can be considered a biomarker for TAD.

Although Wanamaker and colleagues ${ }^{11}$ studied dissection patients only-a very exclusive group of patients with aortic disease-our patient cohort reflects the overall TAD patient population. We found no difference in

TABLE 3. Demographic characteristics and risk factors of patients with aortic aneurysms and acute aortic syndromes

\begin{tabular}{|c|c|c|c|c|c|c|c|c|c|}
\hline $\begin{array}{l}\text { Characteristic or } \\
\text { risk factor }\end{array}$ & $\begin{array}{c}\text { Normal branch } \\
\text { pattern }(n=370)\end{array}$ & $\begin{array}{c}\text { BAA } \\
(\mathbf{n}=\mathbf{1 3 7})\end{array}$ & $P$ value* & $\begin{array}{c}\text { ILVA } \\
(\mathbf{n}=\mathbf{3 5})\end{array}$ & $P$ value* & $\begin{array}{c}\text { ARSA } \\
(\mathbf{n}=\mathbf{1 0})\end{array}$ & $P$ value* ${ }^{*}$ & $\begin{array}{l}\text { Total anomalous } \\
\quad(\mathbf{n}=\mathbf{1 8 6})\end{array}$ & $P$ value* \\
\hline Male gender & 68.6 & 62.7 & .914 & 82.9 & .086 & 50 & .301 & 70.4 & .698 \\
\hline Hypertension & 82.3 & 74.3 & .060 & 80 & .817 & 80 & .694 & 75.8 & .090 \\
\hline Hyperlipidemia & 39.5 & 41.9 & .610 & 42.9 & .720 & 30 & .746 & 41.9 & .584 \\
\hline Smoking & 27.7 & 26.5 & .823 & 23.5 & .691 & 10 & .299 & 25.4 & .612 \\
\hline Diabetes & 9.3 & 9.6 & .863 & 14.3 & .365 & 10 & 1.000 & 10.2 & .760 \\
\hline Bicuspid aortic valve & 25.9 & 33.8 & .095 & 37.1 & .164 & 30 & .725 & 33.9 & .058 \\
\hline Age at presentation (y) & $60.1 \pm 13.7$ & $56.7 \pm 13.8$ & .013 & $56.4 \pm 14.9$ & .135 & $56 \pm 17.6$ & .355 & $56.7 \pm 14$ & .007 \\
\hline Age at surgery (y) & $62.4 \pm 12.9$ & $58.5 \pm 13.5$ & .003 & $58.3 \pm 14$ & .085 & $58 \pm 16.2$ & .295 & $58.6 \pm 13.7$ & .002 \\
\hline
\end{tabular}

Values are presented as $\%$ or mean \pm standard deviation. $B A A$, Bovine aortic arch; ILVA, isolated left vertebral artery; ARSA, aberrant right subclavian artery. $*$ Calculated in comparison to patients with normal branch pattern. 
TABLE 4. Demographic characteristics and risk factors in patients with aortic aneurysms

\begin{tabular}{|c|c|c|c|c|c|c|c|c|c|}
\hline $\begin{array}{l}\text { Characteristic or } \\
\text { risk factor }\end{array}$ & $\begin{array}{c}\text { Normal branch } \\
\text { pattern }(n=285)\end{array}$ & $\begin{array}{c}\text { BAA } \\
(\mathbf{n}=\mathbf{1 0 9})\end{array}$ & $P$ value* & $\begin{array}{c}\text { ILVA } \\
(n=26)\end{array}$ & $P$ value* & $\begin{array}{l}\text { ARSA } \\
(\mathbf{n}=9)\end{array}$ & $P$ value* $*$ & $\begin{array}{l}\text { Total anomalous } \\
\qquad(\mathrm{n}=147)\end{array}$ & $P$ value $*$ \\
\hline Male gender & 68.8 & 68.7 & 1.000 & 92.3 & .012 & 44. & .472 & 72.8 & .438 \\
\hline Hypertension & 81.8 & 72.5 & .052 & 73.1 & .297 & 88.9 & 1.000 & 73.5 & .048 \\
\hline Hyperlipidemia & 40.7 & 42.2 & .820 & 46.2 & .678 & 33.3 & .743 & 42.2 & .837 \\
\hline Smoking & 26.7 & 24.8 & .798 & 26.9 & 1.000 & 11.1 & .455 & 24.5 & .645 \\
\hline Diabetes & 9.8 & 7.3 & .559 & 19.2 & .173 & 11.1 & .600 & 9.5 & 1.000 \\
\hline Bicuspid aortic valve & 30.6 & 39.4 & .119 & 50 & .050 & 33.3 & 1.000 & 40.8 & .042 \\
\hline Family history & 34.4 & 29.6 & .401 & 26.9 & .521 & 33.3 & 1.000 & 28.8 & .277 \\
\hline Age at presentation (y) & $59.8 \pm 13.5$ & $56.5 \pm 13.8$ & .030 & $56 \pm 15.7$ & .180 & $58.8 \pm 16$ & .819 & $56.6 \pm 14$ & .023 \\
\hline Age at surgery (y) & $62.3 \pm 12.8$ & $58.7 \pm 13.5$ & .014 & $58.6 \pm 14.9$ & .161 & $59.4 \pm 16.5$ & .510 & $58.8 \pm 13.7$ & .008 \\
\hline
\end{tabular}

Boldface denotes statistical significance. Values are presented as \% or mean \pm standard deviation. BAA, Bovine aortic arch; ILVA, isolated left vertebral artery; ARSA, aberrant right subclavian artery. *Calculated in comparison to patients with normal branch pattern.

frequency of aortic arch anomalies in patients with aortic aneurysms or acute aortic syndromes.

Patients with aortic aneurysms and arch anomalies showed differences in terms of hypertension and presence of bicuspid aortic valve compared with those without arch anomalies. Patients displaying aortic arch variations had a lower rate of hypertension $(73.5 \%$ vs $81.8 \% ; P=.048)$, one of the most important risk factors for TAD. In terms of lower prevalence of risk factors in patients with aortic arch anomalies, Hornick and colleagues ${ }^{10}$ showed a similar pattern with lower rates of hyperlipidemia and tobacco smoking in patients with bovine aortic arch. We believe that these findings may reflect an important influence of aortic arch anomalies on the development of aortic disease; specifically, that arch anomalies are powerful enough to cause aneurysm disease without the help of traditional risk factors. Despite a lower risk factor profile, patients with anomalous aortic branch patterns still seem to experience aortic disease, especially aortic aneurysms.

Rate of bicuspid aortic valves was higher in patients with aortic arch anomalies and aortic aneurysms (40.8\% vs $30.6 \% ; P=.042$ ) and emerged as independent risk factor for TAD in patients with aneurysm. This may reflect a fundamental biologic link between aortic valve morphology and arch morphology (genetic or embryologic).

Surgical treatment of aortic disease being associated with different branching patterns has been described mainly by different case reports, addressing Kommerell diverticuli or bovine aortic arches. ${ }^{15,16,20,21}$ Our data revealed

TABLE 5. Risk factors in patients with aortic aneurysms

\begin{tabular}{lccc}
\hline \multicolumn{1}{c}{ Risk factor } & Odds ratio & $\begin{array}{c}\mathbf{9 5} \% \text { Confidence } \\
\text { interval }\end{array}$ & $\boldsymbol{P}$ value \\
\hline Hypertension & 0.609 & $0.371-1.001$ & .050 \\
Bicuspid aortic valve & 1.519 & $1.02-2.308$ & .049 \\
Hyperlipidemia & 1.165 & $0.76-1.785$ & .484 \\
Diabetes & 1.058 & $0.523-2.140$ & .875 \\
Smoking & 0.866 & $0.541-1.387$ & .549 \\
Gender & 0.810 & $0.517-1.269$ & .357 \\
\hline
\end{tabular}

higher rates of concomitant intervention of the aortic arch in patients with aneurysm who display anomalous branch patterns. Especially patients with a bovine aortic arch undergo hemiarch replacement more frequently than patients without aortic arch anomalies (46\% vs $34.6 \%$; $P=.023)$. Our finding that arch surgery was necessary more frequently in patients with arch anomalies (Table 6) may reflect fundamental alterations in the aortic wall from the time of embryogenesis. ${ }^{22}$

It is important to be aware of arch anomalies at the time of arch replacement because there can be considerable influence on cannulation and perfusion strategies, as well as on the reconstruction itself. Aberrant right subclavian artery mitigates against right axillary cannulation. An isolated left vertebral artery makes for a difficult direct reimplantation of this fragile artery. On the other hand, the presence of bovine aortic arch facilitates right and left brain perfusion with a single cannula. Anomalous aortic branch patterns may fundamentally influence the aortic arch, requiring higher rates of direct arch interventions.

\section{Limitations of the Study}

For a control group, we decided to summate published data on aortic arch patterns in the general population. The 4 studies that were taken into account for the control group reflect patients from 4 different countries in Europe. We believe these large studies were directly pertinent and surpassed any local control group that we might identify.

The control studies did not screen to exclude thoracic aortic aneurysm; however, because thoracic aortic aneurysm is quite rare in the general population, contamination of the control group by patients with aneurysm is quite unlikely to be a significant factor. ${ }^{23}$

Another very specific limitation has to do with the fact that the study by Natsis and colleagues ${ }^{8}$ assessed arch anatomy by angiography, whereas the other studies (and our own data) used computed tomography scan. There is a 
TABLE 6. Surgical procedures

\begin{tabular}{|c|c|c|c|c|c|c|c|c|c|}
\hline Procedure & $\begin{array}{c}\text { Normal branch } \\
\text { pattern }(n=370)\end{array}$ & $\begin{array}{c}\text { BAA } \\
(\mathbf{n}=\mathbf{1 3 7})\end{array}$ & $P$ value* & $\begin{array}{c}\text { ILVA } \\
(\mathbf{n}=\mathbf{3 5})\end{array}$ & $P$ value* & $\begin{array}{c}\text { ARSA } \\
(\mathbf{n}=\mathbf{1 0})\end{array}$ & $P$ value $*$ & $\begin{array}{c}\text { Total anomalous } \\
\quad(\mathrm{n}=\mathbf{1 8 6})\end{array}$ & $P$ value* \\
\hline Ascending replacement & 54.6 & 46 & .109 & 42.9 & .216 & 60 & 1.000 & 46.8 & .088 \\
\hline Ascending arch & 34.6 & 46 & .023 & 40 & .579 & 20 & .504 & 43 & .063 \\
\hline Arch only & 1.1 & 0.7 & 1.000 & 0 & 1.000 & 10 & .126 & 1.1 & 1.000 \\
\hline Arch + descending & 0.5 & 0.7 & 1.000 & 5.7 & .039 & 0 & 1.000 & 1.6 & .340 \\
\hline Descending replacement & 4.3 & 2.9 & .611 & 8.6 & .220 & 10 & .372 & 4.3 & 1.000 \\
\hline Thoracoabdominal replacement & 4.9 & 3.6 & .639 & 2.9 & 1.000 & 30 & 1.000 & 3.2 & .508 \\
\hline Arch intervention & 36.3 & 47.5 & .025 & 45.7 & .277 & 0 & 1.000 & 45.7 & .034 \\
\hline
\end{tabular}

Boldface denotes statistical significance. Values are presented as n (\%). BAA, Bovine aortic arch; ILVA, isolated left vertebral artery; ARSA, aberrant right subclavian artery. *Calculated in comparison to patients with normal branch pattern.

possibility of lack of total congruence between modalities, especially for the more subjective bovine determination rather than the dichotomous aberrant left vertebral artery or anomalous right subclavian artery.

Because the main focus of our study was to examine prevalence of aortic branch patterns in patients with TAD, data on aortic growth rates are not presented and aortic sizes were only calculated for patients with aortic dissection. The effect of aortic arch anomalies on aortic size and growth would be an appropriate topic for a future study.

\section{CONCLUSIONS}

Our results strongly associate the presence of anomalous aortic arch branching patterns with TAD. Prevalence of aortic arch variations is elevated in patients with TAD when compared with the general population. We conclude that abnormal aortic arch patterns should no longer be considered clinically irrelevant or benign variations; instead, clinicians need to be aware of the presence of aortic branching variations as a potential anatomic marker for future development of TAD.

\section{Conflict of Interest Statement}

Authors have nothing to disclose with regard to commercial support.

\section{References}

1. National Center for Injury Prevention and Control. WISQARS leading causes of death reports, 1999-2007. http://webappa.cdc.gov/sasweb/ncipc/leadcaus 10. html. Accessed February 21, 2015.

2. Fedak PW, Verma S, David TE, Leask RL, Weisel RD, Butany J. Clinical and pathophysiological implications of a bicuspid aortic valve. Circulation. 2002; 106:900-4.

3. Albornoz G, Coady MA, Roberts M, Davies RR, Tranquilli M, Rizzo JA, et al. Familial thoracic aortic aneurysms and dissections-incidence, modes of inheritance, and phenotypic patterns. Ann Thorac Surg. 2006;82:1400-5.

4. Kuzmik GA, Feldman M, Tranquilli M, Rizzo JA, Johnson M, Elefteriades JA. Concurrent intracranial and thoracic aortic aneurysms. Am J Cardiol. 2010; 105:417-20.

5. Celikyay ZR, Koner AE, Celikyay F, Deniz C, Acu B, Firat MM. Frequency and imaging findings of variations in human aortic arch anatomy based on multidetector computed tomography data. Clin Imaging. 2013;37:1011-9.

6. Jakanani GC, Adair W. Frequency of variations in aortic arch anatomy depicted on multidetector CT. Clin Radiol. 2010;65:481-7.
7. Muller M, Schmitz BL, Pauls S, Schick M, Röhrer S, Kapapa T, et al. Variations of the aortic arch — a study on the most common branching patterns. Acta Radiol. 2011;52:738-42.

8. Natsis KI, Tsitouridis IA, Didagelos MV, Fillipidis AA, Vlasis KG, Tsikaras PD. Anatomical variations in the branches of the human aortic arch in 633 angiographies: clinical significance and literature review. Surg Radiol Anat. 2009;31:319-23.

9. Berko NS, Jain VR, Godelman A, Stein EG, Ghosh S, Haramati LB. Variants and anomalies of thoracic vasculature on computed tomographic angiography in adults. J Comput Assist Tomogr. 2009;33:523-8.

10. Hornick M, Moomiaie R, Mojibian H, Ziganshin B, Almuwaqqat Z, Lee ES, et al. 'Bovine' aortic arch—a marker for thoracic aortic disease. Cardiology. 2012;123: 116-24.

11. Wanamaker KM, Amadi CC, Mueller JS, Moraca RJ. Incidence of aortic arch anomalies in patients with thoracic aortic dissections. J Card Surg. 2013;28:151-4.

12. Davies RR, Gallo A, Coady MA, Tellides G, Botta DM, Burke B, et al. Novel measurement of relative aortic size predicts rupture of thoracic aortic aneurysms. Ann Thorac Surg. 2006;81:169-77.

13. Rizzo JA, Chen J, Fang H, Ziganshin BA, Elefteriades JA. Statistical challenges in identifying risk factors for aortic disease. Aorta. 2014;2:45-55.

14. Malone CD, Urbania TH, Crook SE, Hope MD. Bovine aortic arch: a novel association with thoracic aortic dilation. Clin Radiol. 2012;67:28-31.

15. Nasir A, Jadoon M, Ellis PK, Graham AN. Kommerell's diverticulum, risk factor for aortic dissection. J Card Surg. 2009;24:463.

16. Ebner L, Huber A, Christe A. Right aortic arch and Kommerell's diverticulum associated with acute aortic dissection and pericardial tamponade. Acta Radiol Short Rep. 2013;2. 2047981613476283.

17. Stone WM, Ricotta JJ 2nd, Fowl RJ, Garg N, Bower TC, Money SR. Contemporary management of aberrant right subclavian arteries. Ann Vasc Surg. 2011;25: 508-14.

18. Moise MA, Hsu V, Braslow B, Woo YJ. Innominate artery transection in the setting of a bovine arch. J Thorac Cardiovasc Surg. 2004;128:632-4.

19. Wells P, Estrera A. Blunt traumatic innominate pseudoaneurysm and left common carotid occlusion with an associated bovine aortic arch. J Thoracic Cardiovasc Surg. 2005;130:928-9.

20. Choi SY, Jin U, Suh JH, Kim YH. Chronic post-traumatic pseudoaneurysm of the innominate artery with an associated bovine aortic arch resulting in airway obstruction. Eur J Cardiothorac Surg. 2008;34:669.

21. Kaul P, Javangula K, Ganti S, Balaji S, Sivananthan M, Gough M, et al. Continuous selective bilateral antegrade cerebral perfusion through anomalous innominate artery for repair of root, ascending aortic and arch aneurysm-challenges, vagaries and opportunities of bovine arch variant anatomy and review of literature. Perfusion. 2009;24:121-33.

22. Nelson ML, Sparks CD. Unusual aortic arch variation: distal origin of common carotid arteries. Clin Anat. 2001;14:62-5.

23. Stein LS, Elefteriades JA. Epidemiology and natural history of thoracoabdominal aortic aneurysms. In: Chiesa R, Zangrillo O, Alfieri G, Melissano G, Coselli JS, eds. Aortic surgery and anesthesia: How to do it IV: Thoracoabdominal aorta: Surgical and anesthetic management. Italy: Springer; 2011: 25-32.

Key Words: Aortic arch branching variations, thoracic aortic disease, biomarker 